\title{
Colorectal Perforation After Anorectal Manometry for Low Anterior Resection Syndrome
}

\author{
Kyung Ha Lee, Ji Yeon Kim, Young Hoon Sul ${ }^{1}$ \\ Department of Surgery, Chungnam National University Hospital, Daejeon; ${ }^{1}$ Department of Trauma Surgery, Chungbuk National University \\ Hospital, Cheongju, Korea
}

We experienced 3 cases of manometry-induced colon perforation. A 75-year-old man (case 1) underwent anorectal manometry (ARM) 3 years after radiotherapy for prostate cancer and a laparoscopic intersphincteric resection for rectal cancer. A 70-year-old man (case 2) underwent ARM 3 months after conventional neoadjuvant chemoradiotherapy and a laparoscopic low anterior resection for rectal cancer. A 78-year-old man (case 3) underwent ARM 2 months after a laparoscopic intersphincteric resection for rectal cancer. In all cases, a colon perforation with fecal peritonitis occurred. All were treated successfully using prompt and active operations and were discharged without any complications. ARM with a balloon, as a measure of rectal compliance, should be performed 2 months or longer after surgery. If a perforation occurs, prompt and active surgical intervention is necessary due to the high possibility of extensive fecal peritonitis.

\section{Keywords: Rectal cancer; Low anterior resection syndrome; Anorectal manometry; Colon perforation}

\section{INTRODUCTION}

Anorectal manometry (ARM) provides comprehensive information about the physiologic state of the anal sphincter and rectal sensation for evaluation of fecal incontinence or constipation. It is also used to examine patients with low anterior resection syndrome (LARS), which means fecal incontinence due to anorectal anatomical and physiological changes occurring after a low anterior resection (LAR) for rectal cancer $[1,2]$. As the frequency of sphincter-saving procedures has increased due to technical developments in rectal cancer surgery, the incidence of LARS and the use of ARM have also increased. ARM is generally regarded as a safe procedure, and the literatures evaluating manometry-induced colon perforation and its management is lacking. Although a few cases have been reported, most of them were accompanied by complications or death. We successfully treated three patients ex-

Received: January 26, 2017 - Accepted: April 7, 2017

Correspondence to: Young Hoon Sul, M.D.

Department of Trauma Surgery, Chungbuk National University Hospital, 766

1 Sunhwan-ro, Seowon-gu, Cheongju 28644, Korea

Tel: +82-43-269-7847, Fax: +82-43-269-8809

E-mail: ssulyh@gmail.com

(C) 2017 The Korean Society of Coloproctology

This is an open-access article distributed under the terms of the Creative Commons Attribution NonCommercial License (http://creativecommons.org/licenses/by-nc/4.0) which permits unrestricted noncommercial use, distribution, and reproduction in any medium, provided the original work is properly cited. periencing manometry-induced colon perforation with fecal peritonitis without any complications by using prompt and active operations, and we report those cases so as to provide information on the principles and the general course of management for patients experiencing manometry-induced colon perforation.

\section{CASE REPORTS}

\section{Case 1}

A 75-year-old man underwent a laparoscopic intersphincteric resection for stage I rectal cancer and had a history of pelvic irradiation for prostate cancer. He suffered form incontinence three years after the surgery and underwent ARM. Eighteen hours after the ARM, he visited the Emergency Department complaining of abdominal pain. His blood pressure was $125 / 74 \mathrm{mmHg}$, his heart rate was 75 beats/min, his respiratory rate was 20 breaths/min, and his body temperature was $37.1^{\circ} \mathrm{C}$. His abdomen was rigid, and whole abdominal tenderness and rebound tenderness were noticed. Leukocytosis and elevation of C-reactive protein and procalcitonin were found, but neither metabolic acidosis nor lacticemia. Subphrenic free air was seen on chest radiography and a large colon perforation with pneumatosis intestinalis proximal to a previous anastomosis with extensive fecal spillage and extensive intra- and retro-pneumoperitoneum was found on computed tomography (CT) (Fig. 1). A linear 3-cm-long linear perforation was found $7 \mathrm{~cm}$ above the anastomosis, and a Hartmann opera- 
Volume 33, Number 4, 2017

tion was performed. He was discharged on the 11th postoperative day without any complication.

\section{Case 2}

A 70-year-old man underwent conventional neoadjuvant chemoradiotherapy, a laparoscopic LAR with loop ileostomy, and an ileostomy repair two months after the LAR. He complained of LARS, and ARM was performed 1 month after the ileostomy repair. Three hours after the ARM, he visited the Emergency Room complaining of continuous abdominal pain. His blood pressure was 119/72 $\mathrm{mmHg}$, his heart rate was 67 beats/min, his respiratory rate was 18 breaths/min, and his body temperature was $36.3^{\circ} \mathrm{C}$. His abdomen was rigid, and whole abdominal tenderness and rebound tenderness were noticed. Initial laboratory examination showed no abnormal findings. Pulmonary edema and effusion were found on chest radiography, and abundant fecal material in the colon was noticed on abdominal radiography. Because his abdominal pain was severe and did not improve, CT was performed. A large perforation of the sigmoid colon with extensive fecal spillage was found (Fig. 2). A linear 3-cm-long perforation 5 $\mathrm{cm}$ above the anastomosis was found, and a colon segmental resection with loop sigmoid colostomy was performed. He was discharged on the 16th postoperative day without any complication.

\section{Case 3}

A 78-year-old man underwent a laparoscopic intersphincteric resection with loop ileostomy and an ileostomy repair 2 weeks later. He complained of LARS, and ARM was performed 1 month after the ileostomy repair. Three hours after the ARM, he visited the Emergency Room complaining of abdominal pain and hematochezia. His blood pressure was $119 / 69 \mathrm{mmHg}$, his heart rate was

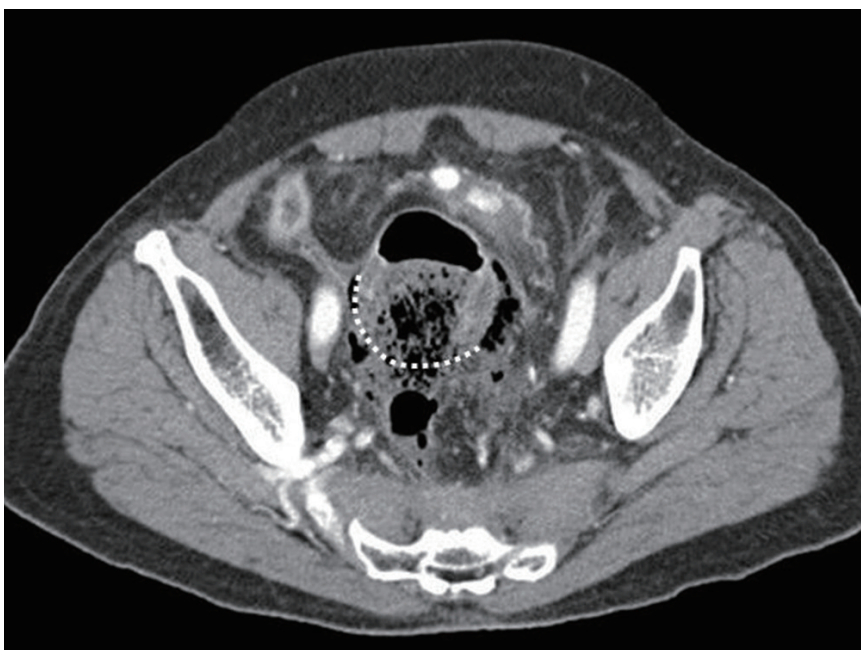

Fig. 1. Computed tomography of the first patient. A large colon perforation with pneumatosis intestinalis proximal to a previous anastomosis with extensive fecal spillage and extensive intra- and retropneumoperitoneum is seen.
76 beats/min, his respiratory rate was 24 breaths/min, and his body temperature was $36.0^{\circ} \mathrm{C}$. His abdomen was rigid, and lower abdominal tenderness and rebound tenderness were noticed. Initial laboratory examination produced no abnormal findings. No free air was seen on chest radiography, and abundant fecal material in colon was noticed on abdominal radiography. CT was performed, and a colon perforation proximal to a previous anastomosis with extensive fecal spillage was found (Fig. 3). A round perforation with a diameter of $4 \mathrm{~cm}$ was found $3 \mathrm{~cm}$ above the anastomosis, and a primary repair with loop transverse colostomy

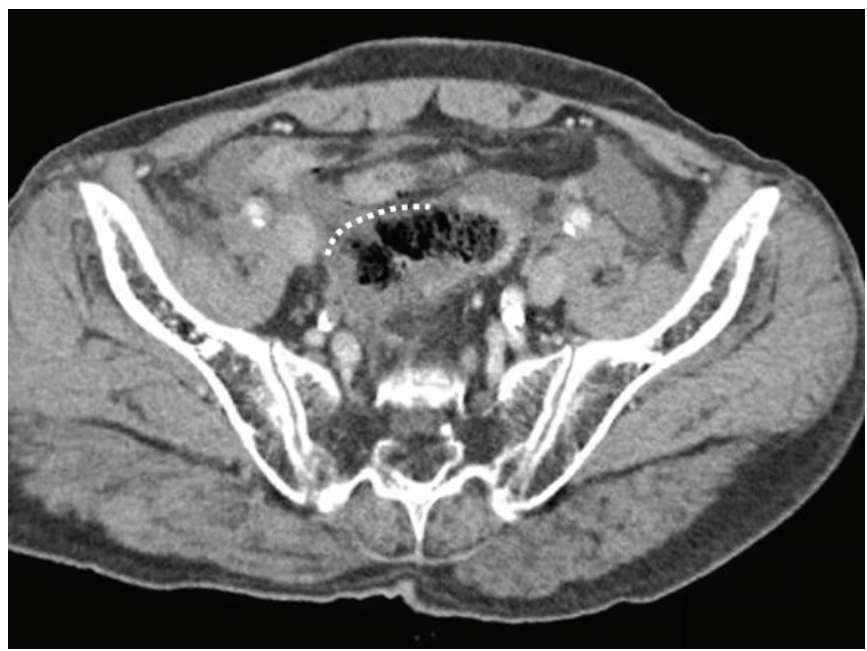

Fig. 2. Computed tomography of the second patient. A large perforation of the sigmoid colon with fecal spillage and complicated fluid collection in the pericolic space is seen.

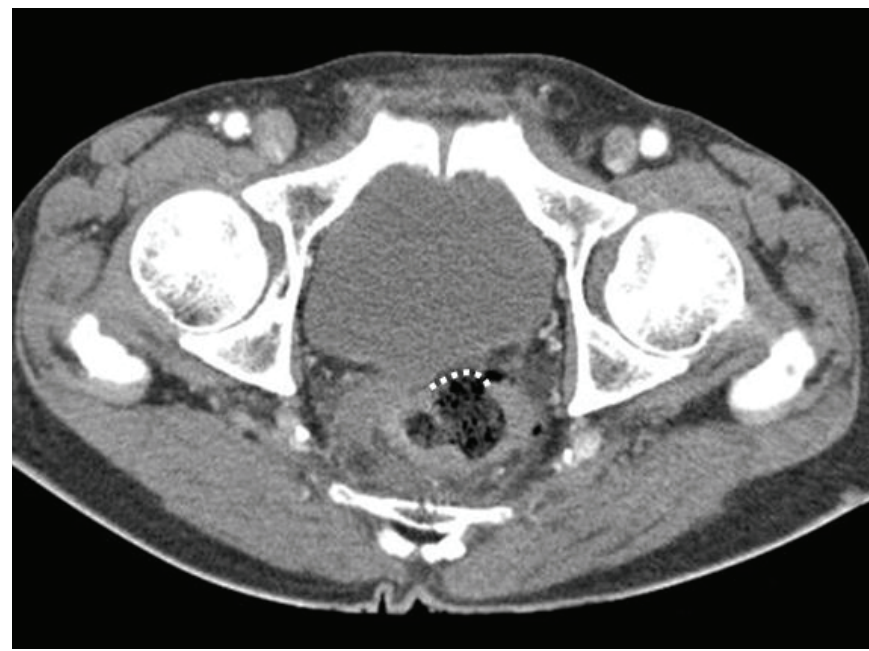

Fig. 3. Computed tomography of the third patient. A focal colon wall defect above the anastomosis site associated with pneumoperitoneum is seen. 
was performed. He was discharged on the 10th postoperative day without any complication.

\section{DISCUSSION}

An iatrogenic colorectal perforation related to ARM is a rare complication. Park et al. [3] reported 2 cases; 1 patient who had undergone a LAR improved after only conservative treatment, but the other who had undergone a LAR following preoperative chemoradiotherapy died immediately after the emergency surgery. Jeong et al. [4] reported 1 case, in which the patient had undergone a LAR following preoperative chemoradiotherapy and was treated by surgery after failure of conservative treatment resulting in colo-cutaneous fistula with abdominal fasciitis. Both authors suggested that manometry should be performed with great care. Park et al. [3] also insisted that the maximum tolerable volume should not be routinely measured for patients who had undergone a restorative proctectomy. In our institute, we perform ARM (Orion Platinum K003367, SRS Medical Systems, Billerica, MA, USA) on patients who present with severe LARS, who are intractable to loperamide, and whose qualities of life are markedly disturbed. Some of those patients are treated with biofeedback therapy. The ARM was initially performed by a colorectal surgeon, but it is now performed by a special nurse of the Outpatient Department, who has several years of recent experience with colorectal surgery. The resting and squeezing pressures are measured by using the stationary and continuous pull-through method and a sensory threshold test including first sensation, desire-to-defecate sensation, and maximal sensation. If the postLAR patient cannot feel the maximal sensation even after $100 \mathrm{~mL}$ of saline is injected into the balloon or the patient complains of anal discomfort or pain, the examination is discontinued. In our cases, the first patient had undergone pelvic irradiation for prostate cancer. He also had chronic constipation combined with fecal incontinence after LAR. Therefore, the rectal wall of this patient was considered to be vulnerable and inelastic because of radiation injury and potential stercoral ulceration. The second patient had also undergone radiotherapy and a LAR. He also underwent manometry 3 months after the surgery. The neorectum was also considered to be vulnerable and inelastic due to radiation injury. The third patient had undergone only a LAR without radiotherapy. However, because the timing of manometry was relatively early, 2 months after the LAR, the neorectum was considered as vulnerable.

In the treatment of an iatrogenic colonic perforation, nonoperative management of the colonic perforation is advocated for patients who are clinically stable and present no evidence of peritonitis [5]. For selected patients with incidental intramural or small retroperitoneal perforations but no evidence of fecal spillage, favorable results have also been reported as the result of conservative treatment consisting of bowel rest combined with total parenteral nutrition, intravenous fluid treatment, and administration of broad-spectrum antibiotics [6]. Especially, when the colonic perforation occurs during a colonoscopy, the bowel content in the colon is minimal because of the bowel preparation, and colonic tissue is generally healthy. Therefore, laparoscopic primary repair or even conservative treatment can be considered [7]. As in the case of colonoscopy-induced colon perforation, in the case of ARM-induced colon perforation, if spillage of bowel content is minimal and the perforation occurs in the mesenteric space, the subsequent symptoms of the patient are usually tolerable, so primary repair or conservative treatment can be considered. However, when a colon perforation occurs after ARM in patients with LARS, the defect is likely to be relatively large; consequently, the fecal spillage tends to be extensive for 2 reasons. First, because their neorectums are relatively vulnerable and inelastic due to postoperative change or radiation injury, the defect is not small, so natural healing is difficult to expect in many cases. Furthermore, an underlying stercoral ulcer due to prolonged intake of loperamide may be present. Second, their neorectums are generally filled with fecal material due to inharmonic defecation in spite of the fecal incontinence associated with LARS. Overflow incontinence related to constipation due to prolonged intake of loperamide may be considerable.

As a result of our experience, especially with these 3 cases, we suggest that ARM with a balloon, as a measure of rectal compliance, be performed two or more months after the surgery. Especially, caution during ballooning is mandatory for irradiated patients regardless of when the examination is performed, even if it is performed several years after the surgery. Also, when a patient with LARS complains of discomfort or pain, especially of the abdomen, a cessation of the examination followed by close observation should be considered. When a perforation occurs, prompt and active surgical intervention is necessary due to high possibility of extensive fecal peritonitis.

\section{CONFLICT OF INTEREST}

No potential conflict of interest relevant to this article was reported.

\section{REFERENCES}

1. Rao SS, Singh S. Clinical utility of colonic and anorectal manometry in chronic constipation. J Clin Gastroenterol 2010;44:597-609.

2. Efthimiadis C, Basdanis G, Zatagias A, Tzeveleki I, Kosmidis C, Karamanlis E, et al. Manometric and clinical evaluation of patients after low anterior resection for rectal cancer. Tech Coloproctol 2004;8 Suppl 1:s205-7.

3. Park JS, Kang SB, Kim DW, Kim NY, Lee KH, Kim YH, et al. Iatrogenic colorectal perforation induced by anorectal manometry: report of two cases after restorative proctectomy for distal rectal cancer. World J Gastroenterol 2007;13:6112-4.

4. Jeong WK, Chung TS, Lim SW, Park JW, Lim SB, Choi HS, et al. 
Rectal perforation after anorectal manometry following preoperative chemoradiotherapy and low anterior resection: report of a cases. J Korean Soc Coloproctol 2008;24:298-301.

5. Gedebou TM, Wong RA, Rappaport WD, Jaffe P, Kahsai D, Hunter GC. Clinical presentation and management of iatrogenic colon perforations. Am J Surg 1996;172:454-7.
6. de Feiter PW, Soeters PB, Dejong CH. Rectal perforations after barium enema: a review. Dis Colon Rectum 2006;49:261-71.

7. Kim J, Lee GJ, Baek JH, Lee WS. Comparison of the surgical outcomes of laparoscopic versus open surgery for colon perforation during colonoscopy. Ann Surg Treat Res 2014;87:139-43. 